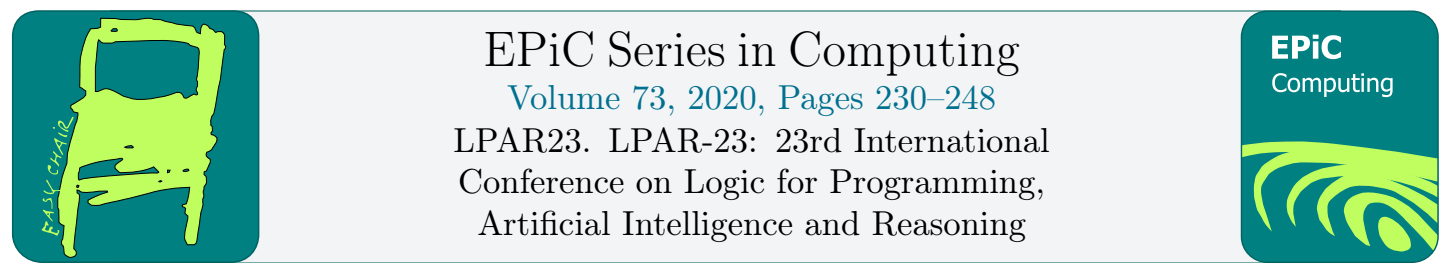

\title{
Deep Reinforcement Learning for Synthesizing Functions in Higher-Order Logic*
}

\author{
Thibault Gauthier \\ Czech Technical University in Prague, Prague, Czech Republic \\ email@thibaultgauthier.fr
}

\begin{abstract}
The paper describes a deep reinforcement learning framework based on self-supervised learning within the proof assistant HOL4. A close interaction between the machine learning modules and the HOL4 library is achieved by the choice of tree neural networks (TNNs) as machine learning models and the internal use of HOL4 terms to represent tree structures of TNNs. Recursive improvement is possible when a task is expressed as a search problem. In this case, a Monte Carlo Tree Search (MCTS) algorithm guided by a TNN can be used to explore the search space and produce better examples for training the next TNN. As an illustration, term synthesis tasks on combinators and Diophantine equations are specified and learned. We achieve a success rate of $65 \%$ on combinator synthesis problems outperforming state-of-the-art ATPs run with their best general set of strategies. We set a precedent for statistically guided synthesis of Diophantine equations by solving $78.5 \%$ of the generated test problems.
\end{abstract}

\section{Introduction}

Improvements in automated theorem provers (ATPs) have been so far predominantly done by inventing new search paradigms such as superposition [13] and SMT [3]. Over the years, developers of these provers have optimized their modules and fine-tuned their parameters. As time progresses, it is becoming evident that more intricate collaboration between search algorithms and intuitive guidance is necessary. ATP developers have frequently manually translated their intuition into guiding heuristics and tested many different parameter combinations. The first success demonstrating the possibility of replacing these heuristics by machine learning guidance has been demonstrated in ITP Hammers [4]. There, feature-based predictors on large interactive theorem prover (ITP) libraries learn to select relevant theorems for a conjecture. This step drastically reduces the search space. As a result, ATPs can prove many conjectures proposed by ITP users. The last landmark that is a major source of inspiration for this paper is the development and success of self-improving neurally guided algorithms in perfect information games [37]. In this work, we adapt such algorithms to theorem proving tasks.

\footnotetext{
*This work has been supported by the European Research Council (ERC) grant AI4REASON no. 649043 under the EU-H2020 programme. We would like thank Josef Urban for his contributions to the first version of this paper.
} 
The hope is that systems using the learning paradigm will eventually improve on and outperform the best heuristically-guided ATPs. We believe that the best design for a solver is one that generalizes across many tasks. One way to achieve this is to minimize the amount of algorithmic bias based on human knowledge of the specific domain. Eventually, given enough examples, the neural architecture might be able to recognize and exploit patterns by itself. For large domains that require vast amount of knowledge and understanding, the number of required examples to capture all the patterns is too large. That is why our experiments are performed on a domain that contains a small number of basic concepts.

Term Synthesis Tasks To test our approach, we choose among theorem proving tasks two term synthesis tasks. In itself, term synthesis is a less commonly explored technique as it is often a less efficient way of exploring a search space as deduction-based methods. This technique is however crucial in inductive theorem proving [10] and in counterexample generators $[7,5]$ as it can be used to provide an induction predicate or a witness.

A term synthesis task can be expressed as proving a theorem of the form $\exists x . P(x)$ with the proof providing a witness for the term $x$. In both our tasks, the theorem can be re-stated as $\exists x . f(x)=y$, where $f$ is an evaluation function specific to the task and $y$ is an image specifying the particular instance that has to be solved. In this light, the aim of the prover is to find an element of the preimage of $y$. This might be hard even if we have an efficient algorithm for $f$ as conjectured by the existence of one-way functions [19].

In the first task, the aim is to construct an untyped SK-combinator which is semantically equal to a $\lambda$-expression in head-normal form. Applications range from better encoding of $\lambda$-expressions in higher-order to first-order translations [34,11] to efficient compilations of functional programming languages to combinator code [24].

In the second task, the aim is to construct a polynomial $p$ whose Diophantine set $D(p)$ is equal to a set of integers $S$. We say that the witness $p$ describes $S$. This process of constructing new and equivalent definitions for $S$ is important during mathematician investigation as it gives alternative point of view for the object $S$. For instance, the set of natural number $\{0,2,4,8,10,12, \ldots\}$ can be describe by the Diophantine equation $k=2 \times x$ defining the concept of even numbers. The Encyclopedia of integer sequences [39] contains entries that can be stated as Diophantine equations (e.g. sequence A001652). Other motivations for investigating Diophantine equations comes from elliptic curve cryptography [2] and number theory [12].

Contributions This paper presents a general framework that lays out the foundations for neurally guided solving of theorem proving tasks. We evaluate the suitability of tree neural networks on two term synthesis tasks. We focus on showing how deep reinforcement learning [41] algorithms can acquire the knowledge necessary to solve such problems through exploratory searches. The framework is integrated in the HOL4 [38] system (see Section 6). The contributions of this paper are: (i) the implementation of TNNs with the associated backpropagation algorithm, (ii) the implementation of a guided Monte Carlo Tree Search (MCTS) algorithm [6] for arbitrarily specified search problems, (iii) the demonstration of continuous self-improvement for a large number of generations, (iv) a comparison with state-of-the-art theorem provers on the task of synthesizing combinators and $(\mathrm{v})$ a formal verification of the solutions in HOL4.

\section{Tree Neural Networks}

In the machine learning field, various kinds of predictors are more suitable for learning various tasks. That is why with new problems come new kinds of predictors. It is particularly true 
for predictors such as neural networks. For maximum learning efficiency, the structure of the problem should be reflected in the structure of the neural network. For example, convolutional neural networks are best for handling pictures as their structure have space invariant properties whereas recurrent networks can handle text better. For our purpose, we have chosen neural networks that are in particular designed to take into account the tree structure of terms and formulas as in [27].

\subsection{Architecture}

Let $\mathbb{O}$ be a set of operators and $\mathbb{T}_{\mathbb{O}}$ be the set of all terms than can be constructed from $\mathbb{O}$. A tree neural network (TNN) is a machine learning model designed to approximate functions from $\mathbb{T}_{\mathbb{Q}} \mapsto \mathbb{R}^{n}$. We define first the structure of the tree neural network and then show how to compute with it. An example is given in Figure 1).

Definition (Tree neural network)

We define a tree neural network to be a set of feed-forward neural networks with $\mathrm{n}$ layers and a tanh activation function for each layer. There is one network for each operator $f$ noted $N(f)$ and one for the head noted $H$. The neural network operator of a function with arity $a$ is to learn a function from $\mathbb{R}^{a \times d}$ to $\mathbb{R}^{d}$ where $d$ is the dimension of the embedding space. And the head network is to approximate a function from $\mathbb{R}^{d}$ to $\mathbb{R}^{n}$. As an optimization for a operator $f$ with arity $0, N(f)$ is defined to be a vector of weights in the embedding space $\mathbb{R}^{d}$ since multiple layers are not needed for learning a constant function.

Computation of the Embeddings Given a TNN, we can now define recursively an embedding function $E: \mathbb{T}_{\mathbb{O}} \mapsto \mathbb{R}^{d}$ by $E\left(f\left(t_{1}, \ldots, t_{a}\right)=_{\operatorname{def}} N(f)\left(E\left(t_{1}\right), \ldots, E\left(t_{a}\right)\right)\right.$ This function produces an internal representation of the terms to be later processed by the head network. This internal representation is often called a thought vector.

Computation of the Output The head network interprets the internal representation and makes the last computations towards the expected result. In particular, it reduces the embedding dimension $d$ to the dimension of the output $n$. The application of a TNN on a term $t$ gives the result $H(E(t))$. It is possible to learn a different objective by replacing only the head of the network. We use this to our advantage in the reinforcement learning experiments where we have the double objective of predicting a policy and a value (see Section 3).

Higher-Order Terms A HOL4 term can be encoded into a first-order term (i.e. a labeled tree) in two steps. First, the function applications $f x$ are rewritten to $\operatorname{apply}(f, x)$ introducing an explicit apply operator. Second, the lambda terms $\lambda x . t$ are substituted with $l a m(x, t)$ using the additional operator lam. For tasks performed on HOL4 terms that are essentially first-order and these encodings are not required.

\section{Deep Reinforcement Learning}

When possible, the deep reinforcement learning approach [41] is preferable to a supervised learning approach for two main reasons. First, an oracle is not required. This means that the algorithm is more general as it does not require a specific oracle for each task and can even learn a task for which nobody knows a good solution. Secondly by decomposing the problem 


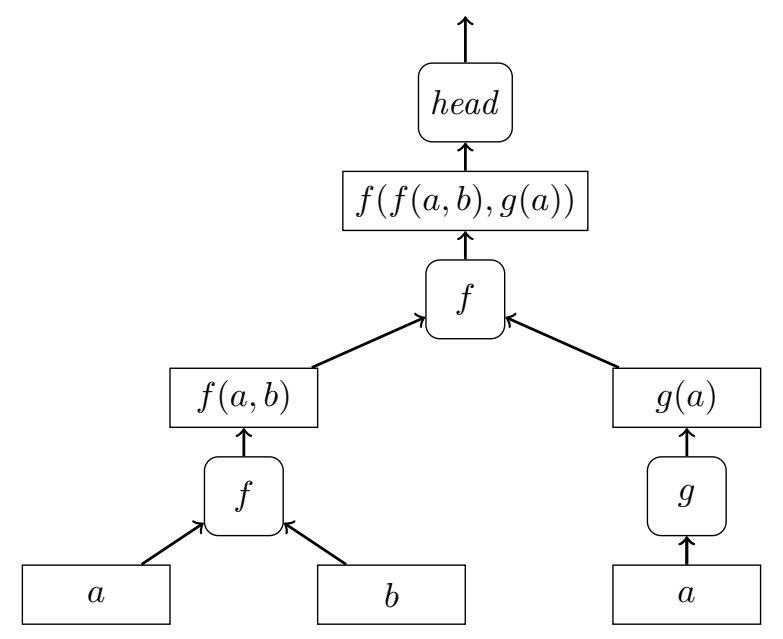

Figure 1: Computation flow during the application of a TNN to the term $f(f(a, b), g(a))$. Rectangles represent embeddings and rounded squares neural networks.

in many steps, the trace of the computation becomes visible which is particularly important if one wants a justification for the final result.

We present here our methodology to achieve deep reinforcement learning through selfsupervised learning. It consists of two phases. During the exploration phase, a search tree is built by the MCTS algorithm from a prior value and prior policy. These priors are updated by looking at the consequence of each action. During the learning phase, a new TNN learns to replicate this improved policy and value. This new TNN will be used to guide the next exploration phase. One iteration of the reinforcement learning loop is called a generation. In the later part of the paper, we refer to the application of the full reinforcement learning loop (alternation of learning phases and exploration phases) as the training of the TNN. Once trained, we judge the performance of the TNN during a final evaluation (Section 6).

Before describing both phases, we define what a search problem is, what the policy and value for a search algorithm are and how they can be combined to guide the search using MCTS.

\subsection{Specification of a Search Problem}

Any task that can be solved in a series of steps with decision points at each step can be used to construct search problems. For example, theorem proving is considered a search task by construction. Other para-proving tasks are harder to view in such a light, such as: programming, conjecturing, making definitions, re-factoring. Here, a search task is described as a single-player perfect information game.

Definition (Search problem)

A search problem is an oriented graph.

The nodes are a set of states $\mathbb{S}$ with particular labels for: a starting state $s_{0} \in \mathbb{S}$, a subset of winning states $\mathbb{W} \subset \mathbb{S}$, and a subset of losing states $\mathbb{L} \subset \mathbb{S}$.

The oriented edges are labeled by a finite set of moves $\mathbb{M}$. The transition function $T: \mathbb{S} \times \mathbb{M} \mapsto \mathbb{S}$ returns the state reached by making a move from the input state. To solve the problem, an 
algorithm needs to find a path $p$ from the starting state to a winning state avoiding the losing states. An end state is a state that is either winning or losing.

Policy and Value A policy $P$ is a function from $\mathbb{S}$ to $[0,1]^{\text {cardinal }(\mathbb{M})}$ that assigns to each state $s$ a real number for each move. It is intended to be a probability which indicates the percentage of times each move should be explored at each state. An impossible move is given a policy score of 0 and thus is never selected during MCTS.

A value $V$ is a function from $\mathbb{S}$ to the interval $[0,1] . V(s)$ is used as an estimate of how likely the search algorithm is to complete the task. Therefore, the function needs to respect these additional constraints: a value of 1 for winning states, and a value of 0 for losing states.

\subsection{Monte Carlo Tree Search}

An in-depth explanation of the Monte Carlo Tree Search algorithm is given in [6]. This search algorithm strikes a good balance between exploration of uncertain paths and exploitation of path leading to states with good value $V$. The algorithm was recently improved in [37]. The estimation of the value $V$, which used to be approximated by the proportion of random walks (or roll-outs) to a winning state, is now returned by a deep neural network. Explicitly, this means that we do not perform roll-outs during the node extension steps and instead the learned value $V$ is used to provide the rewards.

A search problem is explored by the MCTS algorithm with the help of a prior policy $P$ and a prior value $V$. The algorithm starts from an initial tree (that we call a root tree) containing an initial state and proceeds to gradually build a search tree. Each iteration of the MCTS loop can be decomposed into three main components: node selection, node extension and backup. One step of this loop usually creates a new node in the search tree unless the node selection reached an end state (winning or losing). The search is stopped after a fixed number of iterations of the loop called the number of simulations or after a fixed time limit. The node selection process is guided by the PUCT formula [1]. We set its exploration coefficient to 2.0 in our experiments. The rewards are calculated as usual with winning state given a reward of 1 and losing states a reward of 0 and the rewards for other states are given by the value $V$.

\subsection{Exploration Phase}

During the exploration phase of the reinforcement learning loop, a full attempt at a solution will rely on multiple calls to the MCTS algorithm. The first call is performed starting from a root tree containing the starting state. After an application of the MCTS algorithm, the constructed tree is used to decide which move to choose. The application of this move to the starting state is a big step. The MCTS algorithm is then restarted on a root tree containing the resulting state. This procedure is repeated until a big step results in an end state or after the number of big steps exceeds a fixed bound. This bound is fixed to be twice the size of an existing solution found during problem generation (Section 4). An attempt is successful if it ends in a winning state.

The decision which big steps to make is taken from the number of visits for each child of the root. During the exploration phase, the move with the highest number of visits is chosen. To encourage exploration during training, a noise is added to the prior policy of the root node. We draw noise from a uniform distribution and thus do not have to choose the parameter of the Dirichlet distribution as in [37]. 
Remark The upper bound is also used to limit the depth of the MCTS calls during training. Since it gives our prover some problem specific knowledge about the limits of the search space and also prevents it from finding longer solutions, the bound is not enforced during the final evaluations on the testing sets.

Problem Selection It is computationally expensive to make attempts on all 2000 problems from the training set (see Section 5). That is why we select a subset of 200 problems. As we aim to make the dataset of examples balanced, we select 100 positive problems and 100 negative problems. A problem is positive if it was attempted previously and solved in the last attempt and negative otherwise. On top of that, we try to focus the selection on problems away from problems that are too hard or too easy. To estimate how easy and hard a problem is we look at the list of successes and failures of the algorithm on this problem. We count the number of successes (respectively failures) in a row starting from the end of the list on a positive (respectively negative) problem. The inverse of this number is higher for a problem that has just shifted from negative to positive or conversely. This score is normalized into a probability distribution across all the positive (respectively negative) examples. The selected positive (respectively negative) problems are drawn from this distribution.

\subsection{Learning Phase}

After each big step of the exploration phase, we collect an example consisting of an input term representing a search state associated with an improved policy and an improved value for this search state. More precisely, the example is constructed from statistics of the root of the search tree (the root changes after each big step). The input of the example is the term representing the state of the root. The improved policy for this example is computed by dividing the number of visits for each child by the total number of visits for all children. The improved value is the average of the values of all the nodes in the tree with the value of an end state counted as many times as it has been visited. The new example is added to a dataset of training examples. This dataset is then used to train the TNNs in future generations. When the number of examples in the dataset reaches $x$, older examples are discarded whenever newer examples are added so that the number of examples never exceeds $x$. This number is called the size of the window and is set to 200,000 in our experiments.

The TNN learns to imitate the collected policy and value examples by following the batch gradient descent algorithm [30]. In our implementation, we rely on a mean square error loss and update the weights using backpropagation. Furthermore, $P$ and $V$ are learned simultaneously by a double-headed TNN. In other words, the learned term embeddings are shared during the learning of the two objectives.

Remark The states (or their term representation) in the policy and value dataset differs from the dataset of problems. Indeed, problems are starting states whereas policies and values are extracted for all intermediate states covered by the big steps.

\section{Specification of the Tasks}

In this section, we specify the two synthesis tasks that are used for experiments with our framework. We describe how to express the search problems and provide optimizations to facilitate the learning. 


\subsection{Synthesis of Combinators}

The aim of this task is to find a SK-combinator $c$ such that $c x_{1} x_{2} \ldots x_{n} \rightarrow h^{\prime}\left[x_{1}, x_{2}, \ldots, x_{n}\right]$ where $h^{\prime}$ is a higher-order term only composed of the functions $x_{1}, x_{2}, \ldots, x_{n}$ and $\rightarrow$ is rewrite relation given by the term rewrite system $\{S x y z \rightarrow(x z)(y z), K x y \rightarrow x\}$, A combinator is by definition said to normalize if has a normal form. This unique normal form can always be obtained using the left-outermost strategy. Since having a solution $w$ to the problem implies that the combinator $w x_{1} x_{2} \ldots x_{n}$ normalizes and therefore $w$ normalizes too and its normal form $w^{\prime}$ is also a solution. Thus, we can limit the search to SK-combinators in normal form.

To synthesize combinators we introduce a meta-variable $X$ as a place holder at positions of to-be-constructed subterms. The starting state is a couple of the partially synthesized term $X$ and $h^{\prime}\left[x_{1}, x_{2}, \ldots, x_{n}\right]$. A move consists oFf applying one the following five rewrite rules to the first occurrence of $X$ in the first component of the state:

$$
X \rightarrow S, X \rightarrow S X, X \rightarrow S X X, X \rightarrow K, X \rightarrow K X
$$

This system is exactly producing all the combinators in normal forms. It prevents the creation of any redex by limiting the number of arguments of $S$ and $K$. A state is winning if the synthesized witness $w$ applies to $x_{1} x_{2} \ldots x_{n}$ rewrites to the normal form $h^{\prime}\left[x_{1}, x_{2}, \ldots, x_{n}\right]$, which is determined in practice by applying the left-outermost rewrite strategy. We remove occurrences of $X$ in $w$ before testing for the winning condition. A state is losing if the synthesized witness $w$ does not contain $X$ and is not a solution.

TNN Representation of the State To make its decision between the five moves and evaluate the current state, the TNN receives components of the state as first-order terms by tagging each operator with its local arity and merge their embeddings using a concatenation network. For instance, the combinator $S(K S) K$ is encoded as $s_{2}\left(k_{1}\left(s_{0}\right), k_{0}\right)$. Compared with the apply encoding, the advantage is that we can represent each combinator with arity greater than zero as a neural network instead of an embedding in our TNN architecture. The drawback is that the learning is split between operators of different arities.

Complexity For synthesizing a solution of size 20, an upper bound for the search space of our algorithm is $5^{20} \approx 9.5 \times 10^{13}$ as there are 5 possible moves. Since no moves are allowed from end states, there are more precisely at most $4.9 \times 10^{11}$ states with a partially synthesized combinator of size less than 20 .

\subsection{Synthesis of Diophantine Equations}

The aim of this task is given a particular set $S$ to find a polynomial whose Diophantine set is equal to $S$. In the following description and for the experiments, we limit the range of polynomials to the ones with one parameter $k$ and three existential variables $x, y, z$. The Diophantine set $D(p)$ of the polynomial $p$ is defined by $D(p)={ }_{\text {def }}\{k \mid \exists x y z \cdot p(k, x, y, z)=0\}$. To make the computation of $D(p)$ tractable for any polynomial, the domain of interpretation of the variables and operators is changed to $\mathbb{Z} / 16 \mathbb{Z}$.

The polynomials considered for synthesis are normalized polynomials. They are expressed as a sum of monomials. Internally, each monomial is represented as a list of integers. The first element of the list is the coefficient, and the remaining elements are the exponents of the variables $k, x, y$ and $z$. A polynomial is a list of monomials and its size is the sum of the length of its monomials (represented as lists). For instance, the monomial $k^{2} x^{3}+2 y^{4}$ is internally 
represented as $[[1,2,3],[2,0,0,4]]$. Since multiplication is associative and commutative (AC), variables are ordered alphabetically in the monomial. Since addition is AC, monomials are sorted by comparing their list of exponents using the lexicographic order.

Our starting state consists of the empty polynomial and an enumeration $S$. To synthesize a polynomial we rely on two types of moves. The first one is to start constructing the next monomial by choosing its coefficient. The second one is to choose an exponent of the next variable in the current monomial. In practice, we limit the maximum value of an exponent to 4 and the number of monomials per polynomial to 5 . A state $(w, S)$ is winning if the polynomial $w$ defines the set $S$.

TNN Representation of the State Computing the embedding of a state $(w, S)$ relies on a neural network operator for merging the embeddings of $w$ and $S$. The set $S$ is encoded as a list of 16 real numbers. The $i^{t h}$ element of this list is 1 if $i \in S$ and -1 otherwise. The polynomial $w$ is represented using a tree structure with operators + and $\times$ and we have learnable embeddings for each variable and each coefficient. To compress the representation further, variables with their exponent (e.g. $x^{3}$ ) are represented as a single learnable embedding instead of a tree.

Complexity An approximation measure for the size of the search space is the number of polynomials in normal form. This number is $\frac{\left(16 \times 5^{4}\right)^{5}}{5 !} \approx 8.3 * 10^{17}$.

\section{Datasets}

In all our tasks, our algorithms require a training set in order to learn the task at hand. In a reinforcement learning setting, a training problem does not come with its solution as in supervised learning, thus problem-solving knowledge cannot be obtained by memorization and has to be acquired through search. Still, we also create an independent testing set to further estimate the generalization abilities of the algorithm on problems not seen during training. Even in the context of reinforcement learning, the ability of TNNs to learn a task is heavily influenced by the quality of the training examples. The following objectives should guide the generation of the training set: a large and diverse enough set of input terms, a uniform distribution of output classes and a gradual increase in difficulty.

For both tasks, problems are generated iteratively in the same way. At the start, the set of problems $\mathbb{P}$ is empty. At each step, a random witness $w$ (polynomial or combinator) is produced and we compute its image $f(w)$. If the image does not have the desired form, then $\mathbb{P}$ remains unchanged. If the image does not exist in $\mathbb{P}$, we add the problem represented by $f(w)$ and its solution $w$ to the problem set. If the image already exists in the set and the witness is smaller than the previous one for this image, then we replace the previous solution by the new one. If it is bigger, then $\mathbb{P}$ remains unchanged. We repeat this process until we have 2200 distinct problems. This set of problems is split randomly into a training set of 2000 problems and testing set of 200 problems. We use the generated solutions to estimate the difficulty of the problems and bound the number of big steps during training. The generated solutions are not revealed to any other part of the algorithm. In particular, no information about these solutions is used during the final evaluation on the test set.

To generate a random combinator, we pick randomly a size between 1 and 20 and then draw uniformly at random from the set of normal form SK-combinators of that size. Generating this set becomes too computationally expensive for a size greater than 10, thus we rely on a top-down generation that exactly simulates the process. It works by selecting the top operator 


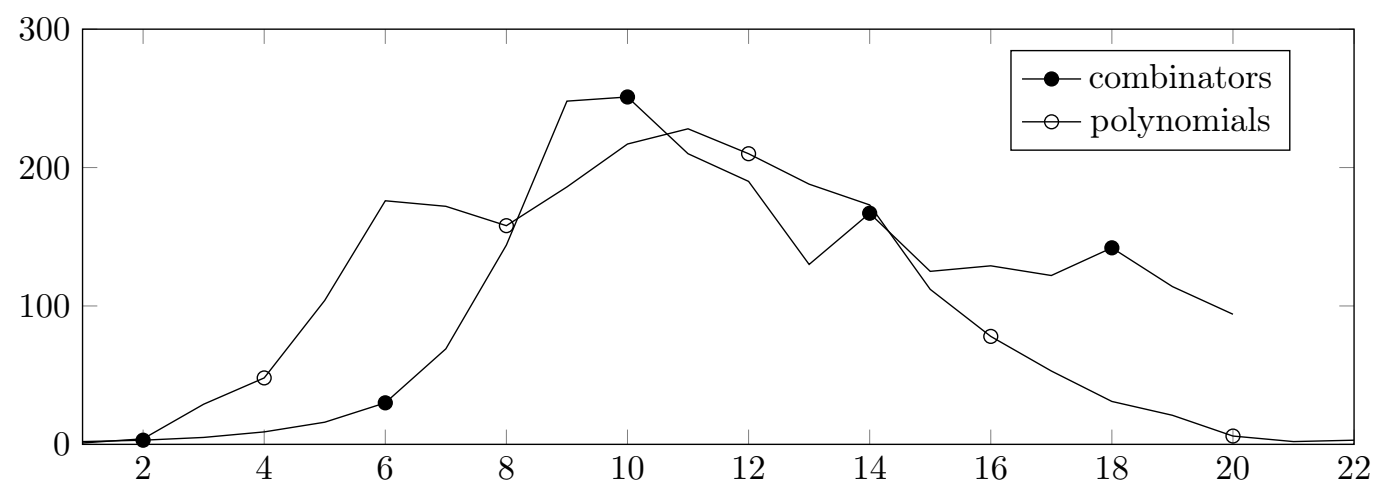

Figure 2: Number (y) of problems a generated solution of size (x)

and the size of its arguments according to their frequencies which can be computed much more efficiently. To generate a random polynomial, we first select a number of monomials in $[|1,5|]$. Then for each monomial, we choose a number of variables in $[|0,4|]$, a leading coefficient in $[|1,15|]$ and an exponent in $[|0,4|]$ for each variable.

Figure 2 shows the distributions of the problems according to their difficulty. There, the size of the smallest generated solution is used as a measure of difficulty for each problem.

\section{Results}

The following experiments demonstrate how the reinforcement learning framework is able to gradually learn each task by recursive self-improvement. We first analyze the progress made during training, Then, we compare our method with alternatives during evaluation. Finally, we analyze the distribution of solutions produced to gain some insight into what has been learned.

Replicability The code for the framework and the experiments are available in the repository for HOL4 ${ }^{1}$. Although the code is available on top of the master branch, to be able to import the provided HOL4 datasets of combinator problems, one needs to switch to the commit bcd916d1251cced25f45c90e316021d0fd8818e9 as the format for exporting terms was changed.

The specification of each task is implemented in the examples/Al_tasks directory. The underlying framework shared by both tasks is located in the src/Al directory. The file examples/AI_tasks/README explains how to reproduce the experiments. The datasets can be downloaded from our repository ${ }^{2}$.

Parameters The parameters of the TNN used during our experiments are an embedding dimension of size 16, one fully connected layer per operator and two fully connected layers for the policy head and the value head. The schedule of the learning phase consists of 10 epochs on a maximum of 200,000 examples and a learning rate of 0.02 .

\footnotetext{
${ }^{1}$ https://github.com/HOL-Theorem-Prover/HOL

${ }^{2}$ https://github.com/barakeel/synthesis_datasets
} 


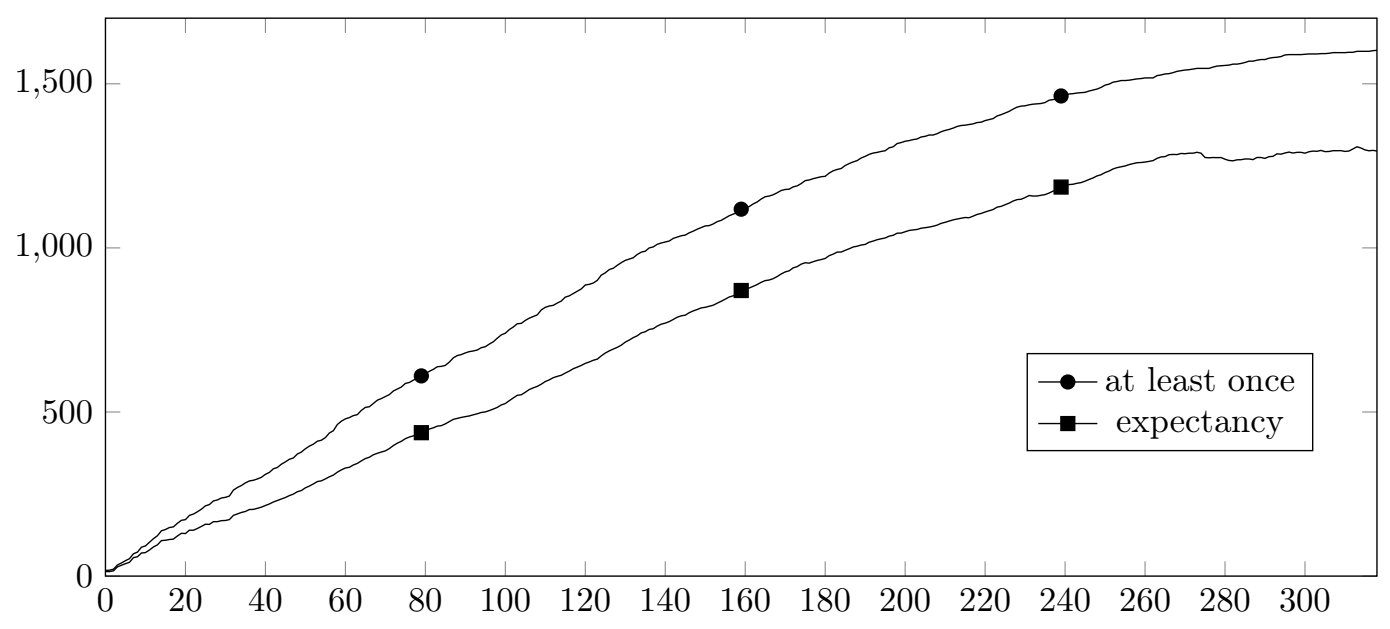

Figure 3: Number $\mathrm{y}$ of training problems solved after generation $\mathrm{x}$

\subsection{Combinators}

Experiments on combinators rely on an instance of the MCTS algorithm given by the specification from Section 4.1 noted $\mathrm{MCTS}_{\text {combin }}$.

Training Figure 3 shows the number of problems solved at least once by $\mathrm{MCTS}_{\text {combin }}$ (run with multiple big steps) over 318 generations. To give a better view of the progress of the algorithm, we also show the number of problems that we expect the algorithm to be able to solve. This number is obtained by computing the frequency at which it solves each problem on the last five tries and summing up the frequencies across all problems. This graph shows that little improvement occurs after generation 270. The discrepancy between the two lines also indicates that our TNN is not able to memorize perfectly the previously discovered proofs. One solution to these issues would be to increase the dimension of the embeddings. The trade-off is that it will slow down quadratically the speed of inference and may lead to a weaker generalization ability if applied without proper regularization. All but 100 examples are attempted before generation 34. At this point, only 135 solutions are found. This is to be compared to the total of 1599 solutions found by the end of the training. The oldest examples retrieved from searches are discarded after generation 64 as their number surpasses 200,000. After each generation, we save the weights of the trained TNN. In the following evaluation, we use the weights of the TNN from generation 318 .

Evaluation During the final evaluation, we run each prover on one CPU for 60 seconds on each problem. Their success rates are presented in Table 1. The MCTS combin $_{\text {algorithm is }}$ run without noise and for as many simulations as the time limit allows. In particular, we do not apply any big steps (non-backtrackable steps) as they were mainly introduced to produce training examples for states appearing deeper in the search. By comparing the results of $\mathrm{MCTS}_{\text {combin }}$ on the training and testing set, we observe that it generalizes well although not perfectly to unseen data. Our machine learning guided can then be compared with the uniform strategy (approximating breadth-first search) where each branch is explored with the same probability. Since the uniform strategy does not call the TNN, it can perform on average more 


\begin{tabular}{llcc}
\hline Prover & Strategy & Train (2000) & Test (200) \\
\hline E-prover 2.4 [36] & auto & 38.80 & 36.0 \\
& auto-schedule & 50.35 & 48.5 \\
Vampire 4.2.2 [29] & default & 4.15 & 3.5 \\
& CASC mode & 63.45 & 62.0 \\
MCTS $_{\text {combin }}$ & uniform & 27.65 & 27.0 \\
& TNN-guided & 72.70 & 65.0 \\
\hline
\end{tabular}

Table 1: Percentage of problems solved within 60 seconds

than twice as many simulations $(414,306$ vs 196,000$)$ in 60 seconds. Even with this advantage, the trained algorithm outperforms it significantly.

To compare our algorithm with state-of-the-art automated theorem provers, the synthesis problem is stated using an existential first-order formula for the conjecture. As an example, the problem for synthesizing a combinator equal to the head normal form $\lambda v_{1} v_{2} v_{3} . v_{3}$ can be expressed in the TPTP [40] format (an input format for ATPs) as:

fof $(\operatorname{axS}, \operatorname{axiom}, \quad ![X, Y, Z]:(a(a(a(s, X), Y), Z)=a(a(X, Z), a(Y, Z))))$.

fof $(\operatorname{axK}, \operatorname{axiom}, \quad ! \mathrm{X}, \mathrm{Y}]:(\mathrm{a}(\mathrm{a}(\mathrm{k}, \mathrm{X}), \mathrm{Y})=\mathrm{X}))$.

fof (conjecture, conjecture, ? [Vc]: ! [V1, V2, V3]: $(\mathrm{a}(\mathrm{a}(\mathrm{a}(\mathrm{Vc}, \mathrm{V} 1), \mathrm{V} 2), \mathrm{V} 3)=\mathrm{V} 3))$.

Even using their more advanced set of strategies (auto-schedule for E-prover and CASC mode for Vampire), the success rates of ATPs are less than the trained MCTS combin $_{\text {on }}$ both the training and testing sets. It is worth mentioning that the ATPs and our systems work in a very different way on the synthesis tasks. In our system, there is a synthesis part guided by our TNN and a checking part performed by a deterministic normalization algorithm. In contrast, the ATPs are searching for a proof by applying the rules of their calculus. They essentially deduce intermediate lemmas (clauses) to obtain the proof. As a comparison, the number of generated clauses by E-prover on average on this dataset in 60 seconds is about three million. One advantage of the approaches of ATP is that they might be able to split the synthesis problem is smaller ones by finding an independent part of the head normal form. The trade-off is that with more actions available, smarter strategies are needed to reduce the search space. We believe that combining both approaches, i.e. learning to guide searches on the ATP calculus, would certainly lead to further gains. Finally, our algorithm naturally provides a synthesized witness but it may be harder to extract such witness from an ATP proof. Therefore, we can now analyze the witnesses provided as solutions during the final evaluation.

Examples The $\lambda$-abstraction $\lambda f x y$. fyx gives a semantic description of the $C$ combinator commonly used in functional programming. The solution $S(S(K S)(S(K K) S))(K K)$ proposed by our algorithm is not equivalent to the solution $S(B B S)(K K)$ given by Schönfinkel [35] with $B=S(K S) K$. Its normal form $S(S(K(S(K S) K)) S)(K K)$ is different. The witness synthesized by $\operatorname{MCTS}_{\text {combin }}$ for $\lambda x y z . x y(x y)(y(x y)(x y(y(x y)))) z$ is the largest among the solutions. This combinator is $S(S(S(K(S(S(K S)))) K) S)(S(S(S(S K K))))$.

Analysis of the Solutions In Table 2, we observe patterns in the subterms of the solutions by measuring how frequently they occur. This only gives us a very rough understanding of what the TNN has learned since the decisions made by the TNN are context-dependent. The combinator $S$ occurs about twice as often as $K$. More interestingly, the combinator $S K K$ 


\begin{tabular}{lccccccc}
\hline Subterm & $S$ & $K$ & $S S$ & $S K$ & $K S$ & $S(K S)$ & $S K K$ \\
Occurrences & 11187 & 5114 & 1883 & 1082 & 710 & 709 & 585 \\
\hline Subterm & $S S K$ & $K(S S)$ & $S(K(S S))$ & $S(S K K)$ & $S(S S)$ & $S S(S K)$ & $S(S S K)$ \\
Occurrences & 370 & 305 & 305 & 273 & 245 & 204 & 183 \\
\hline Subterm & $K K$ & $S(K K)$ & $S(K S) K$ & $S(S(S K K))$ & $S(K S) S$ & $S(S(K S) K)$ & \\
Occurrences & 173 & 165 & 153 & 144 & 138 & 135 & \\
\hline
\end{tabular}

Table 2: Number of occurrences of the 20 most frequent subterms that are part of the 1584 combinator solutions of the 2200 combinator problems

occurs 585 times whereas the combinator $S K S$ which as the same effect (i.e. the identity) only 39 times. Having strong preferences between equivalent choices is beneficial as it avoids duplicating searches. However, how this preference was acquired is still to be determined. The combinator $B$ appears in fourth position among the 40 combinators in normal form of size four showing its importance as a building block for combinator synthesis.

\subsection{Diophantine Equations}

Experiments on Diophantine equations rely on an instance of the MCTS algorithm given by the specification from Section 4.2 noted $\mathrm{MCTS}_{\text {dioph }}$.

Training The evolution of the success rate during training is presented in Figure 4. At the end of the training, the number of problems solved at least once is 1986 out of 2000. However, the expectancy is quite lower showing the same issue in the memorization ability of the TNN as for the combinators. This might be solved by increasing the dimension of the embeddings or by improving the network architecture. The number of examples reaches 200,000 at generation 84 later than in the combinator experiments, indicating that the polynomials synthesized are shorter. In general, the fact that we obtain a much higher success rate in this experiment indicates that a higher branching factor but shallower searches suit our algorithm better. In the final evaluation, we use the weights of the TNN from generation 197.

Evaluation The results of the final evaluation presented in Table 4.2 show a drastic difference between the uniform strategy and the TNN-guided one. Thus, this task produces many patterns that the TNN can detect. This observation is reinforced by the fact that the uniform algorithm performs on average 3 to 4 times more simulations in 60 seconds $(269,193$ vs 79,976$)$ than the TNN-guided one. To create a stronger competitor, we handcraft an evaluation function reflecting our intuition about the problem. An obvious heuristic is to guide the algorithm by how many of the elements of $[|0,15|]$ the current polynomial correctly classifies as a member of the targeted set or not. This number is then divided by 16 to give an evaluation function between 0 and 1. The results are displayed in the heuristic row in Table 4.2. Surprisingly, the heuristically guided search performed worse than the uniform strategy mainly due to an overhead cost for computing the evaluation resulting in 70,233 simulations (on average) per MCTS calls. We do not know of any higher-order theorem prover with support for arithmetic. Finding an encoding of the arithmetic operations and/or the higher-order features, which makes the problems tractable for a targeted ATP, is non-trivial and is not investigated in this paper. That is why we did not compare our results with ATPs on Diophantine equations. 


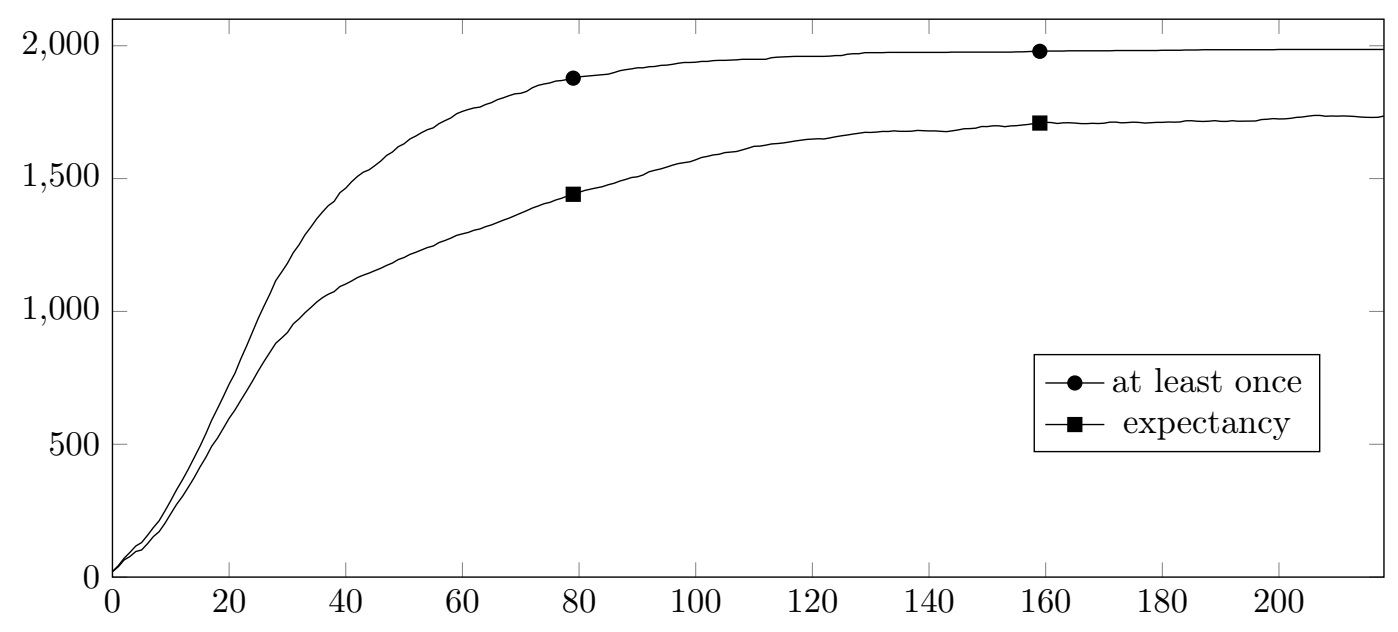

Figure 4: Number y of problems solved after generation $\mathrm{x}$

\begin{tabular}{lcc}
\hline Strategy & Train (2000) & Test (200) \\
\hline uniform & 3.70 & 4.0 \\
heuristic & 3.05 & 0.5 \\
TNN-guided & 77.15 & 78.5 \\
\hline
\end{tabular}

Table 3: Percentage of problems solved in 60 seconds by $\mathrm{MCTS}_{\text {Dioph }}$

Example The solution for the set $\{0,1,3,4,5,9,11,12,13\}$ is the largest among all solutions. The algorithm generated the polynomial $y^{2}+12 x^{4}+7 k+7 k^{2} x^{2} y^{2}+7 k^{2} x^{2} y^{2} z^{2}$.

Analysis of the Solutions The most frequent monomials appearing as part of a polynomial solution are shown in Table 4. The coefficients of those monomials are either even or 7. Only the monomials with coefficient 7 contain an existential variable $x, y$ or $z$. The exponent of an existential variable is always 2 . Following these three patterns exactly limits the search space but might make some problems unsolvable. The two previous examples contain monomials outside of these patterns. This shows that the algorithm is able to deviate from them when necessary.

\begin{tabular}{lcccccccccc}
\hline Monomial & $7 k$ & $7 k^{2} x^{2}$ & $7 k^{2} x^{2} y^{2}$ & $7 k^{2}$ & $7 k x^{2}$ & 7 & 14 & $7 k^{4}$ & $7 k^{4} x^{2}$ & 12 \\
Occurences & 780 & 682 & 640 & 387 & 354 & 329 & 218 & 149 & 144 & 143 \\
\hline Monomial & 8 & $7 k^{3}$ & $14 k$ & $7 k^{2} x^{2} y^{2} z^{2}$ & 4 & 6 & $7 k^{4} x^{2} y^{2}$ & 10 & $10 k$ & 2 \\
Occurences & 118 & 111 & 106 & 92 & 90 & 74 & 72 & 69 & 68 & 61 \\
\hline
\end{tabular}

Table 4: Number of occurrences of the 20 most frequent monomials that are part of the 1700 polynomial solutions of the 2200 problems on Diophantine equations 


\section{Verification}

A final check to the correctness of our algorithm can be made by verifying the solutions produced during evaluation. The verification consists of producing HOL4 theorems stating that each witness $w$ has the desired property $f(w)=c$. The function $f$ is specified by the task and the image $c$ is given in the problem. In the following, we present how to construct a verification procedure for an arbitrary problem/solution pair for the two tasks.

\subsection{Combinators}

First, we express the properties of combinators as HOL4 formulas. There exists an implementation of typed combinators in HOL4 but they cannot be used to represent untyped combinators. For instance, the combinator $S(S K K)(S K K)$ constructed with HOL4 constants is not welltyped. Therefore, we use the apply operator apply of type $\alpha \rightarrow \alpha \rightarrow \alpha$ and the type $\alpha$ for representing the type of combinators. We note $x . y$ the term apply $(x, y)$. Definitions of the free variables $s$ and $k$ representing the combinators $S$ and $K$ are added as a set of assumptions to every problem. From a witness $w$ and a head normal form $h=\lambda x y z \cdot h^{\prime}(x, y, z)$, we prove the theorem:

$$
\{\forall x y z \cdot((s \cdot x) \cdot y) \cdot z=(x \cdot z) \cdot(y \cdot z), \quad \forall x y \cdot(k \cdot x) \cdot y=y\} \vdash \forall x y z \cdot((w \cdot x) \cdot y) \cdot z=h^{\prime}(x, y, z)
$$

A call to the tactic ASM_REWRITE_TAC [] completes the proof. The function COMBIN_PROVE, following the described process, was used to verify the correctness of the 130 solutions found by $\mathrm{MCTS}_{\text {combin }}$ on the test set.

\subsection{Diophantine Equations}

Since problems on Diophantine equations are about sets of natural numbers described by using arithmetic operations, we rely on HOL4 constants and functions from the theories num, arithmetic and pred_set to express that the solution satisfies the problem. We verify that $D(w)$ (the Diophantine set described by the polynomial $w$ ) is the enumeration $\left\{a_{1}, a_{2}, \ldots, a_{i}\right\}$. The equality between the two sets can be expressed in HOL4 as:

$$
\left\{k \mid k<16 \wedge \exists x y z . w\left(k, x_{16}, y_{16}, z_{16}\right) \bmod 16=0\right\}=\left\{a_{1}, a_{2}, \ldots, a_{i}\right\}
$$

For any variable $v$, the shorthand $v_{16}$ stands for $v \bmod 16$. All natural numbers are expressed using the standard HOL4 natural numbers. All variables have the type of HOL4 natural numbers. That is why, in order to reason modulo 16, each existential variable $v$ is replaced by $v \bmod 16$ and the parameter $k$ is bounded by 16 .

The proof starts by considering two predicates $P$ and $Q$ defined by:

$$
P={ }_{\text {def }} \lambda k .\left(\exists x y z . w\left(k, x_{16}, y_{16}, z_{16}\right)=0\right), \quad Q={ }_{\text {def }} \lambda k .\left(k=a_{1} \vee k=a_{2} \vee \ldots \vee k=a_{i}\right)
$$

To verify that these predicates are equivalent on a particular element $k \in[|0,15|]$, we distinguish between two cases. Either the Diophantine equation has a solution and both predicates are true, or it does not admit a solution and both predicates are false. In both cases, ground equations are proven using EQT_ELIM O EVAL where EVAL is a rule (a function that returns a theorem) for evaluating ground expressions. 
Positive case Let us assume that $k \in\left\{a_{1}, a_{2}, \ldots, a_{i}\right\}$. To prove $P k$, we need to prove that $\exists x y z . w\left(k, x_{16}, y_{16}, z_{16}\right) \bmod 16=0$. Therefore, we search for the triple $(a, b, c) \in[|0,15|]^{3}$ for which the ground equation holds. We call the resulting theorem thm_abc. The goal $P k$ can then be closed by applying the tactic:

EXISTS_TAC a THEN EXISTS_TAC b THEN EXISTS_TAC c THEN ACCEPT_TAC thm_abc

$Q k$ is proven by beta-reduction and a call to a decision procedure for ground arithmetic:

CONV_TAC (TOP_DEPTH_CONV BETA_CONV) THEN DECIDE_TAC

Negative case Let us now assume that $k \notin\left\{a_{1}, a_{2}, \ldots, a_{i}\right\}$. To prove $\neg(P k)$, we need to prove that $\forall x y z \cdot w\left(k, x_{16}, y_{16}, z_{16}\right) \bmod 16 \neq 0$. We deduce the following lemma for a predicate $R$ and a variable $v: R 0 \wedge R 1 \wedge \ldots \wedge R 15 \vdash \forall v . R v_{16}$. Using this lemma, we can reconstruct the universally quantified theorems from the proof all possible instantiations:

$$
\begin{aligned}
& \vdash w(k, 0 . .15,0 . .15,0 . .15) \bmod 16 \neq 0 \\
& \vdash \forall z . w\left(k, 0 . .15,0 . .15, z_{16}\right) \bmod 16 \neq 0 \\
& \vdash \forall y z . w\left(k, 0 . .15, y_{16}, z_{16}\right) \bmod 16 \neq 0 \\
& \vdash \forall x y z . w\left(k, x_{16}, y_{16}, z_{16}\right) \bmod 16 \neq 0
\end{aligned}
$$

The notation $\vdash t[0 . .15]$ is a shorthand for $\vdash t[0] \wedge t[1] \wedge \ldots \wedge t[15]$. The proof of $\neg(Q k)$ relies on the same tactic as for proving $Q k$ in the previous case.

By combining the positive and negative cases with some simple propositional reasoning, we obtain equivalences for all $k \in[|0,15|]$. From these equivalences, we prove the following lemma about the bounded sets:

$$
(P 0 \Leftrightarrow Q 0) \wedge \ldots \wedge(P 15 \Leftrightarrow Q 15) \vdash\{k \mid k<16 \wedge P k\}=\{k \mid k<16 \wedge Q k\}
$$

The final step is to convert the set defined by $Q$ into an enumeration by proving the lemma:

$$
\vdash\left\{k \mid k<16 \wedge\left(\lambda k .\left(k=a_{1} \vee k=a_{2} \vee \ldots \vee k=a_{i}\right)\right) k\right\}=\left\{a_{1}, a_{2}, \ldots, a_{i}\right\}
$$

The function DIOPH_PROVE encompassing this process was used to verify the correctness of the 157 solutions found by $\mathrm{MCTS}_{\text {dioph }}$ on the test set.

\section{Related Work}

The related work can be classified into three categories: automation for solving synthesis tasks, machine learning guidance inside ATPs, learning assisted reasoning in ITPs. We present the most promising projects in each category separately. Their description shows how they compare to our approach and influence our methods.

First, synthesizing SK-combinators using machine learning from their defining property has been attempted in [14]. There, they use a genetic algorithm to produce combinators with a fitness function that encompasses various heuristics. By comparison, we essentially try to learn this fitness function from previous search attempts. The bracket abstraction algorithm developed by Schönfinkel [35] can be used to eliminate a variable and by abstracting all variables achieves SK-combinator synthesis. An improvement of the abstraction algorithm using families of combinators is proposed in [28]. A more recent work attempt to solve combinatory logic synthesis problems using a SMT solvers [26]. Matiyasevich proved [32] that every enumerable 
set is a Diophantine set. It is theoretically possible although not practical to extract an algorithm from his proof. The closest attempt at synthesizing Diophantine equations does not rely on statistical learning and is concerned with the synthesis of polynomials over finite Galois fields [21]. If we consider functions to be programs, there is a whole domain of research dedicated to program synthesis. Among these, this approach [20] relies on deep reinforcement learning.

Second, the work that comes closest to achieving our end goal of a competitive learningguided theorem prover is described in [25]. There, a guided MCTS algorithm is trained with reinforcement learning. Its objective is to prove first-order formulas from Mizar [18] problems using a connection-style search [33]. The experiments show that gradual improvement stops on the test set after the fifth generation. This is probably for two reasons: the small number of problems relative to the diversity of the domains considered and the inherent limitations of the feature-based predictor [8] they rely on. Another approach is to modify state-of-the-art ATPs by introducing machine-learned heuristics to influence important choice points in their search algorithms. A major project is the development of ENIGMA [22, 9, 23] which guides given clause selection in E-prover [36]. There, a fast machine learning model is trained to evaluate clauses from their contribution to previous proofs. A significant slowdown detrimental to the success rate of E-prover occurs when trying to replace the fast predictors by deep neural networks [31].

Third, our work aims to ultimately bring more automation to ITP users. Hammers [4] rely on machine learning guided premise selection, translation to first-order and calls to external ATPs to provide powerful push-button automation. An instance of such a system is implemented in HOL4 [15]. Its performance on induction problems is limited by the encoding of the translation. To solve this issue, the tactical prover TacticToe $[16,17]$, also implemented in HOL4, learns to apply tactics extracted from existing proof scripts. It can perform induction on variables when an induction tactic has been defined for the particular inductive type. Yet, it is currently limited by its inability to synthesize terms as arguments of tactics.

\section{Conclusion and Future Work}

Our framework exhibits good performance on the two synthesis tasks exceeding the performance of state-of-the-art ATP on combinators solving $65 \%$ of the test problems. Its success rate reaches $78.5 \%$ on Diophantine equations. Our proposed approach showcases how self-learning can solve a task by gathering examples from exploratory searches. Compared to supervised learning, this self-learning approach does not require the solutions of the problem to be known in advance.

In the future, we intend to test this reinforcement learning framework on many more tasks and test the possibility of joint training [42]. One domain to explore consists of other important tasks on higher-order terms such as beta-reduction or higher-order unification. Another interesting development is to apply the ideas of this paper to the TacticToe framework. A direct application would give the tactical prover the ability to synthesize the terms appearing as arguments of tactic. In general, our framework is able to construct programs and therefore could be adapted to perform tactic synthesis.

\section{References}

[1] David Auger, Adrien Couëtoux, and Olivier Teytaud. Continuous upper confidence trees with polynomial exploration - consistency. In Machine Learning and Knowledge Discovery in Databases - European Conference, ECML PKDD 2013, Prague, Czech Republic, September 23-27, 2013, Proceedings, Part I, pages 194-209, 2013. 
[2] Harald Baier. Efficient algorithms for generating elliptic curves over finite fields suitable for use in cryptography. PhD thesis, Darmstadt University of Technology, Germany, 2002.

[3] Clark W. Barrett, Roberto Sebastiani, Sanjit A. Seshia, and Cesare Tinelli. Satisfiability modulo theories. In Armin Biere, Marijn Heule, Hans van Maaren, and Toby Walsh, editors, Handbook of Satisfiability, volume 185 of Frontiers in Artificial Intelligence and Applications, pages 825-885. IOS Press, 2009.

[4] Jasmin C. Blanchette, Cezary Kaliszyk, Lawrence C. Paulson, and Josef Urban. Hammering towards QED. Journal of Formalized Reasoning, 9(1):101-148, 2016.

[5] Jasmin Christian Blanchette and Tobias Nipkow. Nitpick: A counterexample generator for higherorder logic based on a relational model finder. In Interactive Theorem Proving, First International Conference, ITP 2010, Edinburgh, UK, July 11-14, 2010. Proceedings, pages 131-146, 2010.

[6] C. B. Browne, E. Powley, D. Whitehouse, S. M. Lucas, P. I. Cowling, P. Rohlfshagen, S. Tavener, D. Perez, S. Samothrakis, and S. Colton. A survey of Monte Carlo tree search methods. IEEE Transactions on Computational Intelligence and AI in Games, 4(1):1-43, 2012.

[7] Lukas Bulwahn. The new Quickcheck for Isabelle - random, exhaustive and symbolic testing under one roof. In Certified Programs and Proofs - Second International Conference, CPP 2012, Kyoto, Japan, December 13-15, 2012. Proceedings, pages 92-108, 2012.

[8] Tianqi Chen and Carlos Guestrin. XGBoost: A scalable tree boosting system. In Proceedings of the 22nd ACM SIGKDD International Conference on Knowledge Discovery and Data Mining, San Francisco, CA, USA, August 13-17, 2016, pages 785-794, 2016.

[9] Karel Chvalovský, Jan Jakubuv, Martin Suda, and Josef Urban. ENIGMA-NG: efficient neural and gradient-boosted inference guidance for E. In Automated Deduction - CADE 27 - 27th International Conference on Automated Deduction, Natal, Brazil, August 27-30, 2019, Proceedings, pages 197$215,2019$.

[10] Koen Claessen, Moa Johansson, Dan Rosén, and Nicholas Smallbone. Automating inductive proofs using theory exploration. In Maria Paola Bonacina, editor, Conference on Automated Deduction (CADE), volume 7898 of $L N C S$, pages 392-406. Springer, 2013.

[11] Lukasz Czajka. Improving automation in interactive theorem provers by efficient encoding of lambda-abstractions. In Jeremy Avigad and Adam Chlipala, editors, Proceedings of the 5th ACM SIGPLAN Conference on Certified Programs and Proofs, Saint Petersburg, FL, USA, January 20-22, 2016, pages 49-57. ACM, 2016.

[12] Daniel Duverney. Number Theory: An Elementary Introduction Through Diophantine Problems, volume 4. World Scientific, 2010.

[13] Laurent Fribourg. A superposition oriented theorem prover. Theor. Comput. Sci., 35:129-164, 1985.

[14] Matthias Fuchs. Evolving combinators. In William McCune, editor, Automated Deduction CADE-14, 14th International Conference on Automated Deduction, Townsville, North Queensland, Australia, July 13-17, 1997, Proceedings, volume 1249 of Lecture Notes in Computer Science, pages 416-430. Springer, 1997.

[15] Thibault Gauthier and Cezary Kaliszyk. Premise selection and external provers for HOL4. In Xavier Leroy and Alwen Tiu, editors, Conference on Certified Programs and Proofs (CPP), pages 49-57. ACM, 2015.

[16] Thibault Gauthier, Cezary Kaliszyk, and Josef Urban. TacticToe: Learning to reason with HOL4 tactics. In Thomas Eiter and David Sands, editors, Conference on Logic for Programming, Artificial Intelligence and Reasoning (LPAR), volume 46 of EPiC, pages 125-143. EasyChair, 2017.

[17] Thibault Gauthier, Cezary Kaliszyk, Josef Urban, Ramana Kumar, and Michael Norrish. Learning to prove with tactics. CoRR, 2018.

[18] Adam Grabowski, Artur Korniłowicz, and Adam Naumowicz. Mizar in a nutshell. Journal of Formalized Reasoning, 3(2):153-245, 2010.

[19] Erich Grädel. Definability on finite structures and the existence of one-way functions. Meth. of 
Logic in CS, 1(3):299-314, 1994.

[20] Mohammadhosein Hasanbeig, Natasha Yogananda Jeppu, Alessandro Abate, Tom Melham, and Daniel Kroening. Deepsynth: Program synthesis for automatic task segmentation in deep reinforcement learning. CoRR, abs/1911.10244, 2019.

[21] Abusaleh M. Jabir, Dhiraj K. Pradhan, and Jimson Mathew. An efficient technique for synthesis and optimization of polynomials in $\operatorname{gf}\left(2^{m}\right)$. In Soha Hassoun, editor, 2006 International Conference on Computer-Aided Design, ICCAD 2006, San Jose, CA, USA, November 5-9, 2006, pages 151157. ACM, 2006.

[22] Jan Jakubuv and Josef Urban. ENIGMA: efficient learning-based inference guiding machine. In Intelligent Computer Mathematics - 10th International Conference, CICM 2017, Edinburgh, UK, July 17-21, 2017, Proceedings, pages 292-302, 2017.

[23] Jan Jakubuv and Josef Urban. Hammering Mizar by learning clause guidance. CoRR, abs/1904.01677, 2019.

[24] Mike S. Joy, Victor J. Rayward-Smith, and F. Warren Burton. Efficient combinator code. Comput. Lang., 10(3/4):211-224, 1985.

[25] Cezary Kaliszyk, Josef Urban, Henryk Michalewski, and Miroslav Olsák. Reinforcement learning of theorem proving. In Samy Bengio, Hanna M. Wallach, Hugo Larochelle, Kristen Grauman, Nicolò Cesa-Bianchi, and Roman Garnett, editors, Advances in Neural Information Processing Systems 31: Annual Conference on Neural Information Processing Systems 2018, NeurIPS 2018, 3-8 December 2018, Montréal, Canada, pages 8836-8847, 2018.

[26] Fadil Kallat, Tristan Schäfer, and Anna Vasileva. CLS-SMT: bringing together combinatory logic synthesis and satisfiability modulo theories. In Giselle Reis and Haniel Barbosa, editors, Proceedings Sixth Workshop on Proof eXchange for Theorem Proving, PxTP 2019, Natal, Brazil, August 26, 2019, volume 301 of EPTCS, pages 51-65, 2019.

[27] Eliyahu Kiperwasser and Yoav Goldberg. Easy-first dependency parsing with hierarchical tree LSTMs. TACL, 4:445-461, 2016.

[28] Oleg Kiselyov. \lambda $\lambda$ to ski, semantically - declarative pearl. In John P. Gallagher and Martin Sulzmann, editors, Functional and Logic Programming - 14th International Symposium, FLOPS 2018, Nagoya, Japan, May 9-11, 2018, Proceedings, volume 10818 of Lecture Notes in Computer Science, pages 33-50. Springer, 2018.

[29] Laura Kovács and Andrei Voronkov. First-order theorem proving and vampire. In Natasha Sharygina and Helmut Veith, editors, Computer Aided Verification - 25th International Conference, CAV 2013, Saint Petersburg, Russia, July 13-19, 2013. Proceedings, volume 8044 of Lecture Notes in Computer Science, pages 1-35. Springer, 2013.

[30] Mu Li, Tong Zhang, Yuqiang Chen, and Alexander J. Smola. Efficient mini-batch training for stochastic optimization. In The 20th ACM SIGKDD International Conference on Knowledge Discovery and Data Mining, KDD '14, New York, NY, USA - August 24 - 27, 2014, pages 661670, 2014.

[31] Sarah M. Loos, Geoffrey Irving, Christian Szegedy, and Cezary Kaliszyk. Deep network guided proof search. In LPAR-21, 21st International Conference on Logic for Programming, Artificial Intelligence and Reasoning, Maun, Botswana, May 7-12, 2017, pages 85-105, 2017.

[32] J. V. MATIJASEVIC. Enumerable sets are diophantine. Soviet Math. Dokl., 11:354-358, 1970.

[33] Jens Otten and Wolfgang Bibel. leanCoP: lean connection-based theorem proving. J. Symb. Comput., 36(1-2):139-161, 2003.

[34] Lawrence C. Paulson and Jasmin C. Blanchette. Three years of experience with Sledgehammer, a practical link between automated and interactive theorem provers. In Geoff Sutcliffe, Stephan Schulz, and Eugenia Ternovska, editors, Workshop on the Implementation of Logics (IWIL), volume 2 of EPiC, pages 1-11. EasyChair, 2010. Invited talk.

[35] Moses Schönfinkel. Über die bausteine der mathematischen logik. Mathematische annalen, 92(34):305-316, 1924. 
[36] Stephan Schulz. E - a brainiac theorem prover. AI Communications, 15(2-3):111-126, 2002.

[37] David Silver, Julian Schrittwieser, Karen Simonyan, Ioannis Antonoglou, Aja Huang, Arthur Guez, Thomas Hubert, Lucas Baker, Matthew Lai, Adrian Bolton, Yutian Chen, Timothy Lillicrap, Fan Hui, Laurent Sifre, George van den Driessche, Thore Graepel, and Demis Hassabis. Mastering the game of Go without human knowledge. Nature, 550:354-, 2017.

[38] Konrad Slind and Michael Norrish. A brief overview of HOL4. In Otmane Aït Mohamed, César A. Muñoz, and Sofiène Tahar, editors, Conference on Theorem Proving in Higher Order Logics (TPHOLs), volume 5170 of LNCS, pages 28-32. Springer, 2008.

[39] Neil J. A. Sloane. The on-line encyclopedia of integer sequences. In Manuel Kauers, Manfred Kerber, Robert Miner, and Wolfgang Windsteiger, editors, Towards Mechanized Mathematical Assistants, 14th Symposium, Calculemus 200\%, 6th International Conference, MKM 2007, Hagenberg, Austria, June 27-30, 2007, Proceedings, volume 4573 of Lecture Notes in Computer Science, page 130. Springer, 2007.

[40] Geoff Sutcliffe. The TPTP problem library and associated infrastructure. Journal of Automated Reasoning, 43(4):337-362, 2009.

[41] Richard S. Sutton and Andrew G. Barto. Introduction to Reinforcement Learning. MIT Press, 1st edition, 1998.

[42] Andrew M. Webb, Charles Reynolds, Dan-Andrei Iliescu, Henry W. J. Reeve, Mikel Luján, and Gavin Brown. Joint training of neural network ensembles. CoRR, abs/1902.04422, 2019. 